\title{
Memória Operacional na aprendizagem Matemática: mapeamento teórico de produções brasileiras
}

\author{
Bruna Dorneles Silveira \\ Pontifícia Universidade Católica do Rio Grande do Sul (PUCRS) \\ bruna.dorneles@acad.pucrs.br

\section{Isabel Cristina Machado de Lara} \\ Pontifícia Universidade Católica do Rio Grande do Sul (PUCRS) \\ isabel.lara@pucrs.br
}

\section{Resumo}

Neste artigo apresenta-se um mapeamento teórico de publicações brasileiras com o enfoque na Memória Operacional e suas implicações na Aprendizagem Matemática, com o objetivo de identificar possíveis confluências nas produções selecionadas, comparando aspectos em relação aos objetivos, referências e contribuições sobre esse tema, no contexto da Educação Matemática e na prática docente. Como procedimento metodológico, utiliza-se o mapeamento na perspectiva de Biembengut (2008). Para tanto, selecionou-se uma amostra de dez publicações, no período de 2013 a 2017, por meio da busca no banco de dados do Google Acadêmico, utilizando os termos "Working Memory", "Learning" e "Math", a fim de filtrar as pesquisas a serem analisadas. Com base na análise das produções a partir dos critérios estabelecidos, identificaram-se confluências pertinentes entre as publicações que possibilitaram a emergência de três categorias: intervenções no desenvolvimento das Funções Executivas, avaliações da Memória Operacional, e influências das Funções Executivas para a aprendizagem.

Palavras-chave: Memória Operacional. Aprendizagem. Matemática.

\section{Working Memory in mathematical learning: theoretical mapping of brazilian productions}

\begin{abstract}
This paper presents a theoretical mapping of Brazilian publications focusing on the of Working Memory and its implications in Mathematical Learning, with the objective of identifying possible confluences in selected productions, comparing aspects related to objectives, references and contributions on this theme, in the context of Mathematics Education and teaching practice. As a methodological procedure, the mapping from the perspective of Biembengut (2008) is used. In order to do so, a sample of ten publications were selected, from 2013 to 2017, through Google Scholar database search, using the terms "Working Memory", "Learning" and "Math" to filter the searches to be analyzed. Based on the productions analysis according to established criteria, relevant confluences are identified among the publications that enabled the emergence of three categories: Executive
\end{abstract}


Functions development interventions; Working Memory evaluations; Executive Functions influence for learning.

Keywords: Working Memory. Learning. Math.

\section{Introdução}

O ensino da Matemática, no convívio social do estudante, contribui na medida em que sejam instigadas e exploradas diferentes metodologias e recursos que priorizem a criação de estratégias, a pesquisa, a justificativa, a argumentação, o espírito crítico, e favoreçam a criatividade, o trabalho coletivo, a iniciativa pessoal e a autonomia do desenvolvimento da confiança na própria capacidade de conhecer e enfrentar desafios (SKOVSMOSE, 2004).

Nesse sentido, Skovsmose (2004) afirma que questões sociais e políticas devem ser utilizadas nos processos de ensino e de aprendizagem da Matemática que levem o aluno à reflexão e consequentemente a uma postura crítica.”. O professor ao planejar suas aulas deve considerar o contexto social no qual o estudante está inserido, estimulando-o por meio do uso de estratégias e atividades práticas que oportunizem a reflexão.

A aprendizagem depende da memória e seu uso tem importante função cognitiva; ambas estão intimamente relacionadas (TABAQUIM; RODRIGUES, 2015). De acordo com Izquierdo (2011, p. 11), “[...] memória é a aquisição, a formação, a conservação e a evocação de informações. A aquisição é também chamada de aprendizagem: só se 'grava' aquilo que foi aprendido.".

As funções executivas são um conjunto de processos cognitivos e metacognitivos que possibilitam que o sujeito se envolva e desenvolva no que diz respeito a comportamentos complexos e direcionados, como a realização das atividades escolares por exemplo (DIAS; SEABRA, 2013). O desenvolvimento das Funções Executivas ocorre por meio de diferentes mecanismos, como os de aquisição e de armazenamento. As memórias são evocadas a partir do uso dessas funções. A Memória Operacional, mais mencionada como Memória de Trabalho, é o tipo de memória de curto prazo na qual ocorre um armazenamento temporário de informações, que serão utilizadas de forma imediata para o raciocínio, a resolução de problemas e a elaboração de comportamento (LENT, 2010).

A Memória Operacional exerce muitas funções cerebrais, entre elas, a capacidade de manter as informações em um curto prazo para serem utilizadas por outros processos cognitivos, como, por exemplo, o raciocínio lógico, a manipulação de fórmulas e o planejamento (LENT, 2010). Na Matemática, esses processos são fundamentais para a aprendizagem e, portanto, um bom desenvolvimento da Memória Operacional contribui para a Aprendizagem Matemática. 
Portanto, decidir pesquisar sobre Memória Operacional e Aprendizagem Matemática pressupõe a compreensão de que as Funções Executivas influenciam no desempenho escolar dos estudantes; para isso, manter-se atualizado sobre o que tem sido produzido sobre o tema na área acadêmica faz-se necessário.

Nesse sentido, em razão das contribuições que o entendimento sobre o desenvolvimento da Memória Operacional pode proporcionar para a melhoria do desempenho escolar dos estudantes no ensino da Matemática, emerge a problemática: o que se tem produzido acerca do tema Memória Operacional na Aprendizagem Matemática e quais são as principais contribuições desses estudos para a área da Educação Matemática?

Desse modo, este artigo tem como objetivos: elencar produções acadêmicas na forma de artigo, dissertação ou tese sobre o tema Memória Operacional e sua relação com a Aprendizagem Matemática; comparar aspectos em relação aos objetivos, referências utilizadas, metodologia e análises, com o intuito de compreender os resultados e as contribuições dessas pesquisas sobre o tema para a área da Educação Matemática e para a prática docente.

O processo de seleção dos estudos desenvolveu-se pela busca no banco de periódicos disponibilizados no Google Acadêmico, elaborando, a partir das pesquisas selecionadas, a construção de um mapeamento teórico na perspectiva de Biembengut (2008).

O procedimento metodológico utilizado constitui-se em identificar os estudos existentes com o tema de pesquisa delimitado, no reconhecimento e levantamento dos dados, e na análise dos documentos que possibilitam um amplo conhecimento sobre a área investigada. Um mapa teórico, na concepção de Biembengut (2008, p. 90), “[...] consiste em fazer a revisão na literatura disponível dos conceitos e das definições sobre o tema ou a questão a ser investigada e, a seguir, das pesquisas acadêmicas recentemente desenvolvidas, em especial, nos últimos cinco anos.”. Para a autora, essa metodologia não se baseia na busca pelo conhecimento do senso comum ou da experiência, ao contrário, visa abranger as pesquisas desenvolvidas recentemente, bem como o conhecimento produzido por meio de produções publicadas.

Nesse sentido, o mapeamento teórico auxilia o pesquisador à produção de novas investigações na medida em que reconhece as pesquisas já existentes, não realizando a reprodução do que já foi produzido anteriormente em determinada área de estudo. Assim, o mapeamento teórico restringe-se em três etapas: a primeira envolve os conceitos e definições sobre os termos escolhidos; a segunda apresenta a descrição das pesquisas em estudo por meio da identificação, classificação e organização do mapa; e, a última, no reconhecimento e análise dos dados. 


\section{Conceitos e Definições}

A memória, de acordo com Lent (2010), é a capacidade de armazenar informações que possam ser recuperadas para, posteriormente, ser utilizada por meio de evocações. Por isso, a memória exerce uma importante função executiva. Essas Funções Executivas estão presentes em todos os momentos da vida e são utilizadas cotidianamente para a realização de tarefas (DIAS; SEABRA, 2013). Há vários processos da memória, que se diferem pelo tempo de retenção do armazenamento das informações, podendo ser de curta ou longa duração (LENT, 2010).

A palavra “memória”, para Izquierdo (2011, p. 13-14),

[...] abrange desde os ignotos mecanismos que operam nas placas de meu computador, até a história de cada cidade, país, povo ou civilização, e as memórias individuais dos animais e das pessoas. Mas a palavra 'memória' quer dizer algo diferente em cada caso, porque os mecanismos de sua aquisição, armazenamento e evocação são diferentes.

Cada pessoa possui a sua história e, portanto, a sua memória, pois ao longo da vida acumula fatos, emoções, hábitos, rotinas, que são retidos pela memória e ficam à disposição como uma lembrança instantânea. Uma pessoa que não possui a capacidade de armazenar e evocar as inúmeras informações recebidas tem graves dificuldades no seu desenvolvimento, principalmente, na aprendizagem (COSENZA; GUERRA, 2011). Segundo Lent (2010, p. 644), aprendizagem “[...] é apenas o processo de aquisição das informações que vão ser armazenadas.". Na perspectiva de Tabaquim e Rodrigues (2015, p. 100),

[...] a memória é o cimento mental que liga e interconecta nossas experiências de vida. A vida sem a capacidade de armazenar novas informações ou de evocar experiências previamente estocadas é uma vida em dissolução, sem passado, presente ou futuro mental, sem amarras de si mesma. Não há evidência mais poderosa da importância da memória como possuir o senso de si próprio.

Para as autoras, a memória é essencial para a vida, pois é nela que se armazenam as experiências vivenciadas e cada indivíduo tem as suas próprias experiências, já que o ambiente de desenvolvimento e a possibilidade do uso da linguagem são particulares. Nesse sentido, Tabaquim e Rodrigues (2015, p. 100) corroboram essa perspectiva quando descrevem que "[...] a arquitetura do cérebro de cada pessoa é modificada de forma única.". Entretanto, nem todas as informações recebidas são armazenadas; há um processo de seleção que, às vezes, está ligado à aprendizagem ou a uma misteriosa seleção que pode passar por uma emoção ou um fato relevante (LENT, 2010).

Os estudos sobre Memória Operacional realizadas por Baddeley e Hitch (1974) conceituam a Memória Operacional utilizando-se do Modelo de Multicomponente, que é o mais explorado na literatura, pois apresenta evidências neuropsicológicas. Esse modelo, ao explicar a Memória 
Operacional, inicialmente, tratou de três componentes, sendo um deles dominante, o executivo central, e os outros dois componentes de apoio: alça fonológica e visuoespacial. Posteriormente, o modelo foi aperfeiçoado por Baddeley (2000), no qual o autor adicionou um quarto componente: o retentor episódico.

Para Relvas (2015, p. 24), a neurociência cognitiva “[...] atua nos estudos do pensamento, da aprendizagem, da memória, do planejamento, do uso da linguagem e das diferenças entre memória para eventos específicos e para a execução de habilidades motoras."

A aprendizagem ocorre ao longo da vida. Contudo, a intensidade ou rapidez com que ela ocorre são diferentes em determinados períodos da vida. $\mathrm{O}$ avanço dos estudos realizados pela neurociência cognitiva resulta em valiosas contribuições para a educação. Relvas (2017, p. 64) afirma que, ao aproximar os estudos da Neurociência da escola, “[...] têm-se a possibilidade de realizar uma prática educativa que privilegie qualidade de vida e a melhor compreensão no processo da aprendizagem escolar, para transformar indivíduos de corpos presentes, mentes off-lines em estudantes.” Assim, percebe-se que, ao atrelar os conhecimentos advindos da Neurociência para o âmbito escolar, aproximando essas áreas, criam-se condições para novos estudos em um campo multidisciplinar. Ao abordar a aprendizagem em Matemática, vista como um dos componentes curriculares no qual os estudantes demonstram um baixo rendimento escolar, e que, segundo Lara (2004), é descrita pelos estudantes como uma das disciplinas mais difíceis, vê-se na Neurociência um campo necessário para investigações.

Diante do exposto, abordar os temas Memória Operacional e Aprendizagem Matemática pode auxiliar para compreender, sob o viés das Neurociência, em particular a Neurociência Cognitiva, um pouco mais sobre a aprendizagem nesse componente curricular. Portanto, realizar um mapeamento, a fim de ver como as atuais produções acadêmicas tratam essa temática, pode vir a contribuir para a Educação Matemática.

\section{Mapa de pesquisas acadêmicas}

Apresentam-se, nesta seção, os procedimentos utilizados para a elaboração do mapa de pesquisas acadêmicas, que consiste nos registros da identificação dos artigos e dissertações selecionados, da classificação e organização dos dados, do reconhecimento das principais informações e a compreensão das contribuições de cada produção selecionada. 


\section{Identificação}

No intuito de realizar este mapeamento teórico, utilizaram-se os dados disponibilizados no site de busca Google Acadêmico. A busca por artigos de periódicos e dissertações foi limitada aos últimos cinco anos, de 2013 a 2017. Para explicitar como foi realizada a busca, elaborou-se a Figura1. Um mapa, conforme Biembengut (2008), propõe representar os dados coletados por meio de modelos que servirão como referência para a pesquisa. A partir da expressão “Working Memory”, utilizada a fim de abranger a busca para a nomenclatura estrangeira da expressão "Memória Operacional"1, obtevese como resultado, $556.000^{2}$ documentos escritos em quaisquer idioma. Ao delimitar a busca para as publicações em português foram encontrados 7.040 resultados gerais. Posteriormente, ao adicionar a palavra "Learning" obtiveram-se 1.720 resultados. A busca foi finalizada, utilizando-se a palavra "Math", resultando em 122 publicações. Dessas, foram selecionadas apenas as que continham os termos de busca ou sinônimos, resultando em 33 documentos, entre comunicações breves, artigos, trabalhos de conclusões de cursos de especialização e dissertações.

Figura1 - Processo de seleção das produções

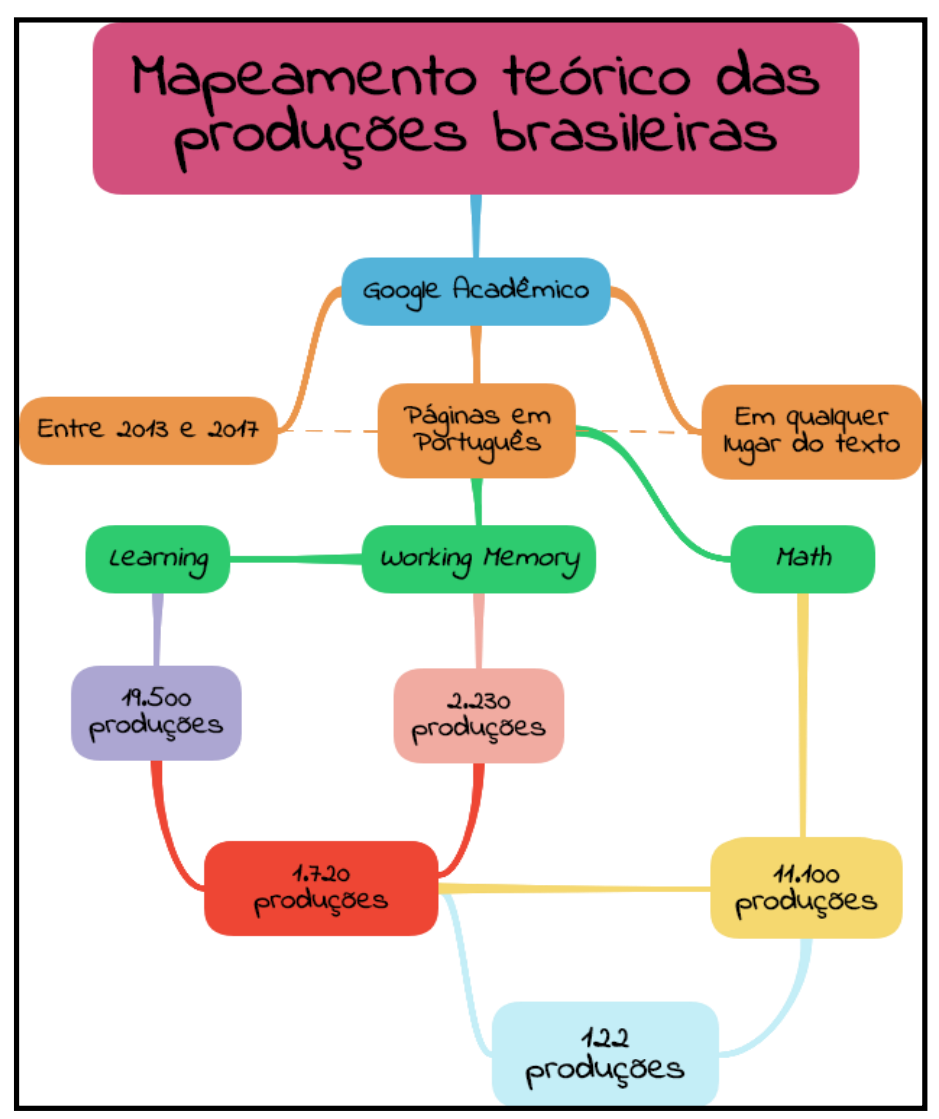

Fonte: Elaborado pelas autoras.

\footnotetext{
${ }^{1}$ Vale sublinhar que optou-se pelos termos em inglês por sua abrangência e por perceber que ao utilizar os termos em língua portuguesa nem todas as produções eram contempladas.

${ }^{2}$ Busca atualizada em 24 de agosto de 2018.
} 
Na concepção de Biembengut (2008), após feita a identificação e organização das produções, refina-se a pesquisa por meio da leitura dos resumos, que ratificarão o vasto conhecimento da área. Finaliza-se, expondo por meio de um mapa a seleção final dos trabalhos de forma a exemplificar a coleta de dados realizada.

Assim, dentre as produções, optou-se por analisar apenas artigos publicados em periódicos brasileiros e dissertações, resultando, após uma breve análise a partir da leitura dos resumos, dez documentos para serem lidos na íntegra, que supostamente possibilitariam identificar relações entre o desenvolvimento da Memória Operacional e Aprendizagem Matemática, conforme apresentado no Quadro 1.

Quadro 1 - Distribuição dos 10 trabalhos selecionados

\begin{tabular}{|c|c|c|c|}
\hline $\begin{array}{c}\text { Tipo de } \\
\text { publicação }\end{array}$ & $\begin{array}{c}\text { Artigos em } \\
\text { periódicos }\end{array}$ & Dissertações & Total \\
\hline 2013 & 1 & 2 & 1 \\
\hline 2014 & 3 & 1 & 5 \\
\hline 2015 & 1 & & 1 \\
\hline 2016 & 1 & & 1 \\
\hline 2017 & 1 & & \\
\hline
\end{tabular}

Fonte: Elaborado pelas autoras com base disponibilizados pelo Google Acadêmico.

A distribuição por ano das produções selecionadas, apresentadas no Quadro 1, é explicitada por meio das quantidades das publicações na área investigada.

\section{Classificação e organização}

Nesta seção, para apresentar as produções selecionadas, elaborou-se o Quadro 2, organizado em ordem cronológica, das dez produções selecionadas, constando a sigla, o ano, autor(es) e o título. As siglas escolhidas foram A $n$ para Artigos e Dn para Dissertação, nas quais $n$ é a numeração conforme a ordem cronológica do tipo de documento.

Quadro 2 - Dados sobre os trabalhos selecionados

\begin{tabular}{|c|c|l|l|}
\hline Sigla & Ano & \multicolumn{1}{|c|}{ Autor(es) } & \multicolumn{1}{|c|}{ Título } \\
\hline A1 & 2013 & $\begin{array}{l}\text { Natália Martins Dias } \\
\text { Alessandra Gotuzo Seabra }\end{array}$ & $\begin{array}{l}\text { Funções executivas: desenvolvimento e } \\
\text { intervenção }\end{array}$ \\
\hline A2 & 2014 & Yasmini Lais Spindler Sperafico & $\begin{array}{l}\text { Intervenção no uso de procedimentos e } \\
\text { estratégias de contagem com alunos dos }\end{array}$ \\
\hline
\end{tabular}




\begin{tabular}{|c|c|c|c|}
\hline & & & $\begin{array}{l}\text { anos iniciais com baixo desempenho em } \\
\text { matemática }\end{array}$ \\
\hline A3 & 2014 & $\begin{array}{l}\text { Gustavo Marcelino Siquara } \\
\text { Maria Virgínia Machado Dazzani } \\
\text { Neander Abreu }\end{array}$ & $\begin{array}{l}\text { Tarefas que avaliam a memória operacional } \\
\text { na infância e adolescência: Uma revisão } \\
\text { sistemática da literatura }\end{array}$ \\
\hline A4 & 2014 & $\begin{array}{l}\text { Luciana Vellinho Corso } \\
\text { Beatriz Vargas Dorneles }\end{array}$ & $\begin{array}{l}\text { A velocidade de processamento e as } \\
\text { dificuldades de aprendizagem na aritmética }\end{array}$ \\
\hline D1 & 2014 & Juliana Schlatter de Lima Ferraz & A Memória na Aprendizagem Matemática \\
\hline D2 & 2014 & Gustavo Marcelino Siquara & $\begin{array}{l}\text { A influência da memória operacional no } \\
\text { desempenho acadêmico em crianças de } 7 \text { a } \\
12 \text { anos de idade }\end{array}$ \\
\hline A5 & 2015 & $\begin{array}{l}\text { Luciana Vellinho Corso } \\
\text { Beatriz Vargas Dorneles }\end{array}$ & $\begin{array}{l}\text { Memória de trabalho, raciocínio lógico e } \\
\text { desempenho em aritmética e leitura }\end{array}$ \\
\hline D3 & 2015 & $\begin{array}{l}\text { Danielle Cristine Borges Piuzana } \\
\text { Barbosa }\end{array}$ & $\begin{array}{l}\text { Intervenção neuropsicológica para manejo } \\
\text { da ansiedade matemática e desenvolvimento } \\
\text { de estratégias metacognitivas }\end{array}$ \\
\hline A6 & 2016 & $\begin{array}{l}\text { Larissa W. Zanella } \\
\text { Nadia C. Valentini }\end{array}$ & $\begin{array}{l}\text { Como funciona a Memória de Trabalho? } \\
\text { Influências na aprendizagem de crianças } \\
\text { com dificuldades de aprendizagem e } \\
\text { crianças com desordem coordenativa } \\
\text { desenvolvimental }\end{array}$ \\
\hline A7 & 2017 & $\begin{array}{l}\text { Hosana Alves Gonçalves } \\
\text { VanisaFanteViapiana } \\
\text { Marcia Santos Sartori } \\
\text { Claudia HofheinzGiacomoni } \\
\text { Lilian Milnitsky Stein } \\
\text { Rochele Paz Fonseca }\end{array}$ & $\begin{array}{l}\text { Funções executivas predizem o } \\
\text { processamento de habilidades básicas de } \\
\text { leitura, escrita e matemática? }\end{array}$ \\
\hline
\end{tabular}

Fonte: Elaborado pelas autoras.

Após a seleção das produções, realizou-se a leitura minuciosa das mesmas por meio da qual elaborou-se uma síntese de cada uma, apresentando, em especial, aspectos como: o problema de pesquisa; os objetivos; contribuições. A partir disso, foi feita uma análise comparativa na tentativa de identificar, quando existisse, pontos convergentes ou divergentes em relação a esses aspectos.

\section{A1: Funções executivas: desenvolvimento e intervenção (2013)}

Diante das constatações que o estudo apresenta em relação ao desenvolvimento das Funções Executivas, as questões de investigação que motivam o estudo são: "1) Qual o desenvolvimento normal das funções executivas? É necessário conhecer o desenvolvimento de tais habilidades para que se possam identificar alterações nesse curso?, e 2) É possível promover o desenvolvimento dessas habilidades? Se sim, como?”. Os principais objetivos dessa produção foram: apresentar/discutir como ocorre o desenvolvimento normal das Funções Executivas; abordar possibilidades de intervenção 
para o desenvolvimento das Funções Executivas; e apresentar pesquisas e programas de intervenção sobre as Funções Executivas.

As contribuições apontam que o desenvolvimento das Funções Executivas, bem como as dificuldades causadas pelo seu subdesenvolvimento, evidenciam a relevância de: conhecer como ocorre o desenvolvimento dessas habilidades e a faixa etária em que acontecem; reconhecer as características das crianças (estudantes) que apresentam habilidades comprometidas; refletir sobre a necessidade das Funções Executivas serem ensinadas, seja no contexto escolar ou clínico; apresentar pesquisas e programas de intervenção do desenvolvimento das Funções Executivas nos currículos escolares e complementares; e instigar a pesquisa dessas intervenções, ampliando o tempo de aplicação e implicações para a aprendizagem.

\section{A2: Intervenção no uso de procedimentos e estratégias de contagem com alunos dos anos iniciais com baixo desempenho em matemática (2014)}

Essa produção não apresenta um problema de pesquisa explícito e teve como objetivo avaliar os efeitos de uma intervenção psicopedagógica nos procedimentos e estratégias de contagem, verificando a evolução desses procedimentos e estratégias e a possibilidade de generalização dos efeitos em outros contextos matemáticos, com um grupo heterogêneo de cinco estudantes.

Destacam-se as seguintes contribuições a partir da leitura minuciosa dessa pesquisa: possibilidades de auxiliar as crianças a desenvolver procedimentos e estratégias de contagem; a evolução no uso de procedimentos e estratégias de contagem por meio de intervenções; a recuperação de fatos da memória a partir das intervenções realizadas. Evidencia que, por meio de intervenções no uso de procedimentos e estratégias de contagem, auxilia os estudantes na realização de tarefas matemáticas e no seu desempenho matemático, bem como na aprendizagem de outros conceitos matemáticos.

\section{A3: Tarefas que avaliam a memória operacional na infância e adolescência: Uma revisão sistemática da literatura (2014)}

Os autores desse artigo não apresentam o problema de pesquisa, porém elencam como objetivos preponderantes: realizar uma revisão de literatura sobre as tarefas utilizadas para avaliar a alçafonológica e o esboço visuoespacial da Memória Operacional na infância e adolescência; analisar os estudos psicométricos referentes as tarefas encontradas utilizadas para avaliar a Memória Operacional. 
A pesquisa aponta contribuições para estudos futuros: dados psicométricos mais refinados para poder afirmar que se está avaliando a Memória Operacional, validação e normatização dos instrumentos no contexto brasileiro, estudos adicionais que investiguem a adequação das tarefas ao modelo de Memória Operacional, seus limites e potenciais quando utilizados com crianças.

\section{A4: A velocidade de processamento e as dificuldades de aprendizagem na aritmética (2014)}

Esse artigo não explicita o problema de pesquisa. No entanto, deu-se ênfase à velocidade de processamento, relacionando tal habilidade com a memória de trabalho e a fluência em fatos aritméticos. Ressalta-se que a velocidade de processamento e a memória de trabalho estão relacionadas e, portanto, afetam o desempenho dos estudantes no desenvolvimento de habilidades matemáticas.

Os resultados da produção mostraram que é inquestionável a necessidade de mais pesquisas que definam o papel da velocidade de processamento e da memória de trabalho vinculada à aprendizagem da matemática, na intervenção preventiva e corretiva das dificuldades apresentadas por estudantes no desempenho aritmético, e, ainda, que o avanço dessas pesquisas podem trazer benefícios para a prática pedagógica, já que há um consenso entre pesquisadores da área da Educação Matemática, da psicologia cognitiva e da neuropsicologia.

\section{D1: A Memória na Aprendizagem Matemática (2014)}

O problema de pesquisa dessa produção foi: "Qual a influência e abrangência da memória na aprendizagem matemática?" Elencam-se como principais objetivos: entender os limites da memória e como ela pode se relacionar com o raciocínio; definir melhor o que é a memória, onde se localiza, quais são as funções cognitivas que trabalham com ela e como ela interfere na aprendizagem de Matemática; estudar a relação entre a memória e o aprendizado e capacidade de raciocínio lógicomatemático.

Os resultados da pesquisa mostraram que o estudo da memória é desvalorizado no âmbito educacional, em que a ênfase é apenas no compreender e não ao lembrar. Notou-se como a ideia de memória é muito mais ampla que a ideia de decorar; por outro lado, o ato de decorar torna as informações consolidadas, levando-as para a memória de longo prazo e, portanto, o professor com esse conhecimento poderia explorar atividades específicas na sala de aula, beneficiando os estudantes no desempenho das atividades na medida que considera-se que o desenvolvimento do componente fonológico presente na memória de trabalho auxilia no raciocínio. 


\section{D2: A influência da memória operacional no desempenho acadêmico em crianças de 7 a 12 anos de idade (2014)}

A questão de investigação dessa pesquisa foi: "Quais são as funções cognitivas chave ou mais fundamentais para a aprendizagem e o eficaz sucesso acadêmico?” Os principais objetivos apresentados foram: investigar se a Memória Operacional é um preditor do desempenho acadêmico em crianças; avaliar as tarefas que são utilizadas na literatura para medir a capacidade da Memória Operacional; identificar se o ambiente socioeconômico pode causar impacto na capacidade da Memória Operacional e no desempenho acadêmico.

Essa pesquisa apresenta contribuições para a literatura, especialmente, no contexto brasileiro, pela carência de estudos nessa área. Os resultados apontam que: a Memória Operacional tem um papel de mediador entre ambiente socioeconômico e o desempenho acadêmico; a Memória Operacional influencia em todas as áreas da aprendizagem e, portanto, afeta as notas escolares dos estudantes em todos os componentes curriculares.

\section{A5: Memória de trabalho, raciocínio lógico e desempenho em aritmética e leitura (2015)}

Nessa produção não foi encontrado um problema de pesquisa. O objetivo foi de descrever o papel que os diferentes componentes da memória de trabalho e raciocínio lógico exercem na variação do desempenho em cálculo aritmético e leitura de alunos do $3^{\circ}$ e $4^{\circ}$ anos do Ensino Fundamental.

Os resultados da pesquisa mostraram correlação entre as medidas de Memória de Trabalho, raciocínio lógico e leitura, além de sugestões de novos estudos nessa área, indicando possibilidades de novas pesquisas que podem trazer contribuições para o entendimento sobre o uso da Memória Operacional e a aprendizagem, bem como o desenvolvimento da intervenção preventiva e corretiva das dificuldades na aritmética e na leitura.

\section{D3: Intervenção neuropsicológica para manejo da ansiedade matemática e desenvolvimento de estratégias metacognitivas (2015)}

Nessa dissertação o problema de pesquisa não foi explicitado. Como objetivo consta testar a eficácia de um programa de intervenção em grupo para redução da ansiedade matemática e desenvolvimento de estratégias metacognitivas de autoeficácia e autorregulação.

Nas considerações foi apontado que as intervenções se mostraram eficazes na redução da ansiedade, especialmente, por se tratar de uma população com um perfil específico de dificuldade de aprendizagem da matemática e alto nível de ansiedade matemática sem comorbidade com outros 
problemas de comportamento, detectados por meio de uma avaliação neuropsicológica. Entretanto, os grupos em que foram realizadas as intervenções, bem como a estudante descrita no estudo de caso, não apresentaram ou não foram observadas melhoras na Memória de Trabalho. Contudo, percebe-se a relevância da pesquisa por apresentar um estudo inédito que não confirma a hipótese de que uma intervenção cognitivo-comportamental para manejo da ansiedade matemática melhora o desempenho em tarefas de memória de trabalho.

\section{A6: Como funciona a Memória de Trabalho? Influências na aprendizagem de crianças com dificuldades de aprendizagem e crianças com desordem coordenativa desenvolvimental (2016)}

Essa produção não apresenta problema de pesquisa. Os principais objetivos dessa pesquisa foram: verificar a função dos componentes da Memória de Trabalho; verificar a influência da Memória de Trabalho no desempenho cognitivo e nas dificuldades de aprendizagem de crianças em idade escolar; verificar as limitações da Memória de Trabalho em crianças com Desordem Coordenativa Desenvolvimental (DCD).

Os resultados para essa revisão de literatura apontam que as dificuldades de aprendizagem estão relacionadas às habilidades da memória de trabalho e, portanto, o desenvolvimento da memória de trabalho é essencial para a aquisição dos processos de aprendizagem. Sugerem-se novas pesquisas que incluam crianças com DCD.

\section{A7: Funções executivas predizem o processamento de habilidades básicas de leitura, escrita e matemática? (2017)}

O problema de investigação não foi encontrado nessa produção. O encaminhamento da pesquisa foi percorrido por meio do seguinte objetivo: analisar o efeito das Funções Executivas - memória de trabalho, inibição, iniciação/velocidade de processamento e flexibilidade cognitiva - na leitura e escrita de palavras e na resolução de operações aritméticas a partir de uma tarefa cujos estímulos abarcam diferentes níveis de complexidade para crianças do primeiro ao nono ano do Ensino Fundamental brasileiro. Identifica-se que houve leitura de diferentes bibliografias, a fim de pontuar os estudos já existentes, usando-as para embasar a discussão dos resultados encontrados.

As contribuições da pesquisa apontam que a memória de trabalho fonológica é um preditor do desempenho de todos os domínios escolares, reforçando a relevância de se considerar o desenvolvimento e potencialidades do processamento executivo na aprendizagem de processos básicos de leitura, escrita e aritmética. Sugere-se que sejam inseridas no contexto escolar, 
especialmente, nos primeiros anos do Ensino Fundamental, intervenções precoce-preventivas das Funções Executivas.

\section{Reconhecimento e análise}

No mapeamento realizado, por meio da leitura apurada das publicações selecionadas, foram identificadas cinco produções de cunho teórico (A1, A3, A4, A6 e D2), quatro pesquisas empíricas (A2, A5, A7 e D3) e uma pesquisa mista (D1), abrangendo a revisão da literatura acerca do tema e os dados empíricos resultantes da aplicação com estudantes.

Com o intuito de reconhecer as confluências, bem como as potencialidades e contribuições dessas pesquisas, que envolveram o tema "Memória Operacional e Aprendizagem Matemática", a leitura minuciosa conduziu à emergência de três categorias: intervenções no desenvolvimento das Funções Executivas, produções A1, A2 e D3; avaliações da Memória Operacional, artigo A3; influências das Funções Executivas para a aprendizagem nas produções A4, A5, A6, A7, D1 e D2.

\section{Intervenções no desenvolvimento das Funções Executivas}

Nas produções A1, A2 e D3, houve confluências no que se refere às intervenções apresentadas: a primeira como pesquisa bibliográfica e as duas últimas pesquisas com estudantes. Por meio da análise, foi possível identificar que as publicações A1 e D3 são direcionadas a psicólogos e neuropsicólogos, assim, endereçadas aos profissionais da saúde em um viés clínico. Já o artigo A1 abrange, ainda, o público da área da educação, em particular, os professores.

A produção A2 dá ênfase aos efeitos das intervenções psicopedagógicas no contexto dos procedimentos e estratégias de contagem e no desempenho em matemática; portanto, voltado à educação.

No estudo detalhado das publicações A1 e D3, houve convergência nos "objetivos", pois ambas apresentam programas de intervenção para o desenvolvimento das Funções Executivas. No artigo A1, as autoras descrevem como ocorre o desenvolvimento das Funções Executivas e indicam possibilidades de intervenção a partir de pesquisas e programas analisados por meio da revisão de literatura. Já na dissertação D3, a intervenção neuropsicológica é explicitada como viabilidade para o manejo da ansiedade matemática e desenvolvimento de estratégias metacognitivas com o escopo de testar a eficácia de um programa de intervenção.

$\mathrm{Na}$ análise do artigo A2, verifica-se que a pesquisadora destaca a intervenção psicopedagógica no uso de procedimentos e estratégias de contagem com estudantes dos anos iniciais 
do Ensino Fundamental com baixo desempenho em matemática com o intuito de avaliar os efeitos das intervenções realizadas por meio de jogos pedagógicos. Nesse sentido, destaca que, a partir das intervenções com o uso de jogos, é possível auxiliar na recuperação da memória para a aprendizagem de Matemática.

\section{Avaliações da Memória Operacional}

A pesquisa A3 discorre sobre os testes que avaliam os componentes da Memória Operacional, enfatizando a necessidade de pesquisas e validação de instrumentos de avaliação da memória no âmbito brasileiro. Os autores, por meio da revisão sistemática da literatura, citam as tarefas utilizadas para avaliar a alça-fonológica e o esboço visuoespacial da Memória Operacional na infância e na adolescência, bem como uma análise minuciosa dos estudos psicométricos referentes a essas tarefas.

No que tange ao público alvo, a produção é direcionada aos profissionais da saúde, ou seja, à área clínica, pois a pesquisa foi publicada na revista Estudos de Psicologia. Foram analisadas nessa pesquisa quarenta e quatro publicações em periódicos científicos nacionais e internacionais que fizeram o uso das atividades que avaliam os componentes da Memória Operacional. Dentre as publicações em estudos nessa produção, a partir da análise detalhada por meio de critérios estabelecidos pelos autores, são descritas vinte diferentes tarefas específicas para avaliar a Memória Operacional alicerçadas no modelo teórico cognitivo. Cabe ressaltar que um dos critérios de exclusão da gama de publicações encontradas foi o de estudos que não investigaram crianças e adolescentes; portanto, todos os artigos com cunho de uma pesquisa empírica.

\section{Influências das Funções Executivas para a aprendizagem}

As pesquisas que integram essa categoria têm como escopo apresentar as influências das Funções Executivas para a aprendizagem. Assim, observa-se que as produções são dirigidas à área da educação enfatizando a relevância desses conhecimentos para os professores. Portanto, identifica-se esse como o primeiro ponto de convergência entre as seis publicações. $\mathrm{Na}$ análise realizada, verificou-se que três pesquisas foram de natureza bibliográfica, duas pesquisas produzidas por meio da aplicação com estudantes e uma com abordagem empírica-bibliográfica.

As confluências nas produções A4, A6, A7 e D2 referem-se aos efeitos das influências no desempenho acadêmico dos estudantes em idade escolar. O artigo A4 disserta sobre as dificuldades de aprendizagem na matemática vinculada à velocidade do processamento e seus efeitos no desempenho escolar. No artigo A6, as pesquisadoras indicam possíveis proximidades das dificuldades de aprendizagem relacionadas com a Memória de Trabalho. Nesse sentido, os objetivos 
da pesquisa intersecionam-se com essas proximidades envolvendo a Memória de Trabalho e suas limitações em crianças com Desordem Coordenativa Desenvolvimental.

Ao analisar os efeitos das Funções Executivas e seus componentes no que se refere à leitura, à escrita e às resoluções das operações aritméticas, as autoras do artigo A7 descrevem as influências resultantes da pesquisa realizada com estudantes do primeiro ao nono ano do Ensino Fundamental por meio da aplicação de tarefas que abarcam diferentes níveis.

Na pesquisa D2, o autor evidencia a necessidade de avaliar as tarefas dispostas na literatura para medir a Memória Operacional por meio da investigação da memória vinculada ao desempenho acadêmico.

A influência da memória para a aprendizagem Matemática foi outra convergência encontrada nessa categoria, identificada nas publicações A5 e D1. O artigo A5 especifica os diferentes componentes da memória de trabalho e o raciocínio lógico, e os efeitos desses componentes no rendimento da Matemática. Quanto à análise feita na pesquisa D1, identifica-se que a autora enfatiza a busca da compreensão dos limites da memória e a sua relação com o raciocínio, bem como a interferência causada na aprendizagem da Matemática.

\section{Considerações finais}

Esta pesquisa foi constituída pelo mapeamento teórico na perspectiva de Biembengut (2008), por meio da análise apurada de uma amostra de dez publicações entre os anos de 2013 e 2017, com a finalidade de reconhecer possíveis confluências nas produções que fazem parte desse escopo. Nas análises feitas, pretendeu-se relacionar os termos utilizados como critério de busca "Working Memory", "Learning" e "Math", e as convergências nas produções selecionadas quanto aos aspectos relacionados aos objetivos, referências e contribuições sobre o tema no contexto da Educação Matemática.

Por meio da leitura integral das pesquisas escolhidas neste mapeamento, foi possível classificá-las e agrupá-las em três categorias, a fim de apresentar de forma compreensível, a partir do enfoque no desenvolvimento da Memória Operacional e das suas implicações na Aprendizagem Matemática, trazendo à tona características peculiares de cada pesquisa por meio da síntese dos seus principais aspectos.

Verifica-se, assim como Lent (2010), que algumas pesquisas utilizam, apenas, o termo memória ao referir-se aos aspectos específicos direcionados à memória operacional, o que ocasiona dificuldade na busca por produções com essa temática; por isso, optou-se pelos termos em inglês, pela abrangência internacional da literatura. 
Em relação à fundamentação teórica das pesquisas, legitimam-se os autores Baddeley e Hitch (1974) e Baddeley (2000) como referências sobre a memória. As produções sugerem e utilizam diferentes bibliografias para embasar teoricamente as pesquisas, porém destaca-se que em todos os estudos os autores mencionados foram referenciados.

Constatou-se, por meio da análise das pesquisas, que apenas quatro produções utilizaram aplicações e estudos empíricos com estudantes; as demais fizeram uso da revisão de literatura. Nesse enfoque, ratifica-se a carência por pesquisas com dados empíricos fundamentados em explicações cognitivas. Para tanto, considera-se que são necessários estudos adicionais que investiguem o desenvolvimento da Memória Operacional e suas implicações para a Aprendizagem Matemática e que esses possibilitem ampliar os conhecimentos hodiernos nessa área.

Salienta-se que esta pesquisa foi um recorte sobre esse tema e que, se fossem outras as publicações selecionadas, outras considerações certamente seriam tecidas. No entanto, as conclusões parciais que aqui são explicitadas chamam a atenção para a importância desse mapeamento acerca do tema em estudo, contribuindo para novas pesquisas que visem explorá-lo e minimizar possíveis lacunas percebidas.

\section{Referências}

BADDELEY, A. D.; HITCH, G. J. Working memory. In: BOWER, G. H. (Org.). The psychology of learning and motivation. London: Academic Press, 1974.

BADDELEY, A. D. Working memory: Theories, models, and controversies. Annual Review of Psychology, 63, 1-29. doi: 10.1146/annurev psych- 120710-100422, 2012.

BIEMBENGUT, M. S. Mapeamento na pesquisa educacional. Editora Ciência Moderna: Rio de Janeiro, 2008.

COSENZA, R. M.; GUERRA, L. B. Neurociência e educação: como o cérebro aprende. Porto Alegre: Artes médicas, 2011.

DIAS, N. M.; SEABRA, A. G. Piafex: Programa de Intervenção em Autorregulação e Funções Executivas. São Paulo: Memnon, 2013.

IZQUIERDO, I. Memória. 2. ed. Porto Alegre: Artmed, 2011.

LARA, I. C. M. Ensino inadequado de matemática. Revista Ciências e Letras, n. 35, p. 141-149, 2004.

LENT, R. Cem bilhões de neurônios?: conceitos fundamentais de neurociência. 2. ed. São Paulo: Editora Atheneu, 2010.

RELVAS, M. P. Neurociências e transtornos de aprendizagem: as múltiplas eficiências para uma educação inclusiva. 6. ed. Rio de Janeiro: Wak Editora, 2015.

. A Neurobiologia da aprendizagem para uma escola humanizadora: observar, investigar e escutar. Rio de Janeiro: Wak Editora, 2017. 
SKOVSMOSE, O. Educação matemática crítica: a questão da democracia. 2. ed. Campinas: Papirus, 2004. 160 p.

TABAQUIM, M. de L. M.; RODRIGUES, S. das D. Memória e aprendizagem. In: CIASCA, S. $M$. et al. Transtornos de aprendizagem: neurociência e interdisciplinaridade. 1. ed. Ribeirão Preto, SP: Book Toy, 2015.

Submetido em outubro de 2018 Aprovado em dezembro de 2018 